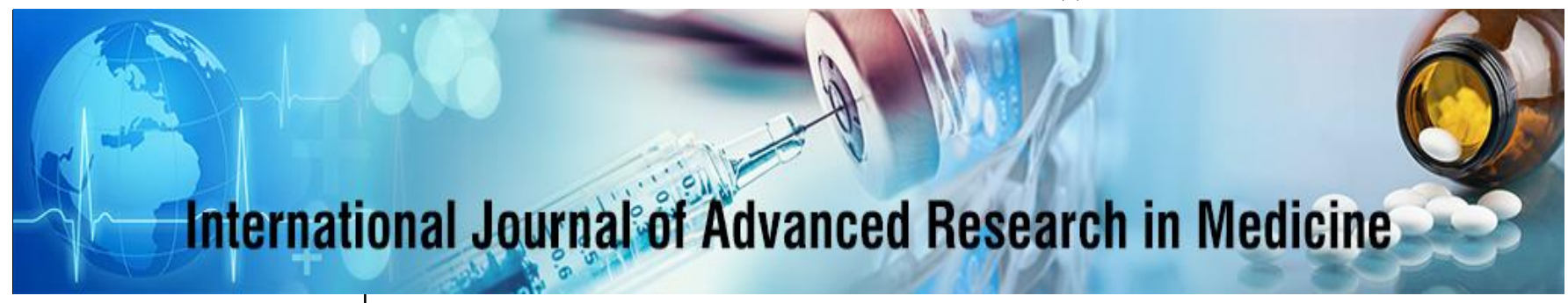

E-ISSN: 2706-9575

P-ISSN: 2706-9567

IJARM 2020; 2(2): 01-04

Received: 03-05-2020

Accepted: 06-06-2020

Dr. Arshdeep S Mann MBBS, Medical Officer at Maan Hospital and Dialysis Center, Bathinda, Punjab, India

Dr. Mandeep Singh Kalsi MBBS, Medical Registrar, Austin Health, Australia

Dr. Beant Singh Maan MBBS, MD, Internal Medicine, Medical Director at Maan Hospital and Dialysis Center, Bathinda, Punjab, India
Corresponding Author: Dr. Arshdeep S Mann MBBS, Medical Officer at Maan Hospital and Dialysis Center, Bathinda, Punjab, India

\section{To determine if digital pulse oximetry is an acceptable screening tool for asymptomatic peripheral vascular disease in type 2 diabetes mellitus: An observational study}

\author{
Dr. Arshdeep S Mann, Dr. Mandeep Singh Kalsi and Dr. Beant Singh \\ Maan
}

DOI: https://doi.org/10.22271/27069567.2020.v2.i2a.39

\begin{abstract}
Aim: to determine if digital pulse oximetry is an acceptable screening tool for asymptomatic peripheral vascular disease in type 2 diabetes mellitus.

Methods: The present analytical cross-sectional study was carried out among 80 patients of type 2 Diabetes Mellitus with asymptomatic PVD were included. The diagnostic accuracy of Ankle-Brachial Index (ABI) and pulse oximetry as a screening tool was compared against Color Doppler ultrasonography as the reference standard.

Results: The prevalence of PVD in the present study was found to be $61.2 \%$. The mean age was 54.16 years, $57.1 \%$ were male, mean duration diabetes was 12.27 years and smoking history was reported in $65.3 \%$ patients. Co-morbidities observed were Hypertension $(59.2 \%)$ followed by Diabetic neuropathy $(48.9 \%)$ and Diabetic Retinopathy $(42.8 \%)$. The sensitivity, specificity, positive predictive value (PPV), negative predicted value (NPV) and accuracy of Pulse oximetry, ABI and Combination to diagnose asymptomatic PVD in diabetics was found to be $(82.45 \%, 77.13 \%, 93.41 \%),(93.21 \%$, $91.46 \%, 97.18 \%),(80.71 \%, 75.60 \%, 90.43 \%),(94.32 \%, 84.34 \%, 98.11 \%)$ and $(86.37 \%, 82.04 \%$, $93.48 \%$ ) respectively.
\end{abstract}

Conclusions: Pulse oximetry is better than ABI for the screening for asymptomatic PVD among diabetics. However, the blend of the two tests has a much higher sensitivity, specificity and accuracy.

Keywords: Accuracy, ABI, PVD, Pulse oximetry

\section{Introduction}

Peripheral Vascular Disease (PVD) is defined as a clinical disorder in which there is stenosis / occlusion of the arteries of the limbs, a common complication of long standing diabetes mellitus ${ }^{[1]}$.

Foot-related disease is the most common cause for hospital admission among the diabetic population and is recognized as the most common cause of non-traumatic lower-limb amputation. The main risk factors for the development of diabetic foot disease are peripheral neuropathy and peripheral arterial occlusive disease ${ }^{[2]}$.

The detection of significant arterial disease is vital to the prevention and treatment of foot disease. The unreliable nature of the symptoms and signs of lower-limb arterial insufficiency in diabetes means that noninvasive tests are essential to achieve effective screening ${ }^{[3,4]}$.

The European Working Group on Critical Leg Ischaemia recommends an additional, noninvasive vascular assessment for patients with diabetes and foot ulceration ${ }^{[5]}$.

Currently, several invasive and non-invasive tests are recommended including pulse palpation, the ankle-brachial index (ABI), pulse-oximetry, color-doppler ultrasonography, arteriography, computed tomographic angiography (CTA) and magnetic resonance angiography (MRA) ${ }^{[6]}$.

The ideal screening test should be inexpensive, non-invasive, accurate, and easy to administer in the physician's office. Imaging modalities like color-doppler ultrasonography, arteriography, Computerized Tomography angiography (CTA) and Magnetic Resonance Angiography (MRA) are accurate, but are very expensive and hence not appropriate for screening purposes. 
Among the less expensive methods used for screening are Ankle-Brachial Index (ABI) and pulse- oximetry ${ }^{[6]}$.

Numerous studies have evaluated and compared the accuracy of different pulse-oximeters over a wide range of clinical conditions. In general, the accuracy of most noninvasive pulse oximeters is acceptable for a wide range of clinical applications.

In India, there is limited access to duplex ultrasonography and other expensive modalities, as it is not available in all areas. If a simple tool like digital pulse-oximetry and ABI will be found to be useful in screening for peripheral artery disease, the complications of peripheral artery obstruction may be addressed at an earlier time. This study aims to determine if digital pulse oximetry is an acceptable screening tool for asymptomatic peripheral vascular disease in type 2 diabetes mellitus

\section{Materials and methods}

The present analytical cross-sectional study was conducted among 80 patients of type 2 Diabetes Mellitus with asymptomatic PVD were included and detailed history was obtained.

\section{Inclusion criteria}

Patients above 30 years of age

Patients pre-diagnosed with Diabetes (as per American Diabetes Association criteria)

Patients with asymptomatic PVD

Those who give informed consent

\section{Exclusion criteria}

1. Those who not give informed consent

2. Patients with history of;

3. Hyper-coagulable states

4. Congestive heart failure

5. Suspected arteritis or collagen vascular diseases

\section{Ethical approval and Informed consent}

The study protocol was reviewed by the Ethical Committee of the Hospital and granted ethical clearance. After explaining the purpose and details of the study, a written informed consent was obtained.

\section{Sample selection}

The sample size was calculated using a prior type of power analysis by G* Power Software Version 3.0.1.0 (Franz Faul, Universitat Kiel, Germany). The minimum sample size was calculated, following these input conditions: power of 0.80 and $P \leq 0.05$ and sample size arrived were 74 participants. The final sample achieved was 80 .

\section{Methodology}

Patients were asked to lie down comfortably in supine posture on the examination couch of the examination room in the department. Finger pulse oximeter was applied first to the right index finger and the left index finger of the patient and the readings noted. This was immediately followed by recording the percentage of oxygen saturation readings in the right great toe and left great toe.

In the next step, patient's lower limbs were elevated one by one in turn to a height of twelve inches off the couch (12" from heel to couch) and pulse-oximeter readings recorded in both the great toes of the patient. After recording the six pulse oximeter readings, measurement of the ankle brachial index was done in all the four limbs.

Diagnosis of PVD was considered when toe saturation is less than finger saturation by $=/>2 \%$ or when foot saturation is decreased by $>2 \%$ in elevated position with abnormal results in at least one limb.

Ankle brachial index was measured with the help of appropriate standard size sphygmomanometer cuffs and a handheld $8 \mathrm{Mhz}$ vascular Doppler probe. The patients were instructed to remain in the supine position. Diagnosis of PVD was considered in case Ankle-Brachial pressure index $(\mathrm{ABI})$ is $<0.9$ in at least one limb.

Color Doppler Ultrasonography was done of the limb involved (i.e. showing abnormal pulse oximetry and /or ABI results) with Doppler Ultrasonography machine (Philips Logiq P6) that uses linear probe of 7.5-12 MHz to evaluate the flow of blood in the vessels. It was considered as the reference standard. A diagnosis of PVD was based on monophasic and biphasic waveforms in any artery by Color Doppler Ultrasonography, and a patient was considered positive for PVD even if any one leg had abnormal results.

\section{Statistical Analysis}

The data was analyzed using SPSS 19 (SPSS Inc. Chicago, IL, USA) Windows software program. Descriptive frequencies were expressed using mean and standard deviation. Sensitivity and specificity were calculated with 95\% confidence interval $(\mathrm{CI})$ where relevant.

\section{Results}

Table 1: prevalence of PVD in the screened population

\begin{tabular}{|c|c|}
\hline PVD & N (\%) \\
\hline Present & $49(61.2)$ \\
\hline Absent & $31(38.8)$ \\
\hline
\end{tabular}

While analyzing the prevalence of peripheral vascular disease was reported in 49 out of 80 patients. The prevalence of PVD in the present study was found to be $61.2 \%$

Table 2: demographic and clinical profile of PVD patients

\begin{tabular}{|c|c|}
\hline Age (years) & $\mathbf{5 4 . 1 6 \pm 3 . 8 1}$ \\
\hline Gender $(\mathrm{M} / \mathrm{F})$ & $28(57.1 \%) / 21(42.9 \%)$ \\
\hline Duration of diabetes & $14.27 \pm 1.18$ \\
\hline Smoking History & $32(65.3 \%)$ \\
\hline Co-morbidities & \\
\hline Hypertension & $29(59.2 \%)$ \\
\hline Diabetic neuropathy & $24(48.9 \%)$ \\
\hline Diabetic Retinopathy & $21(42.8 \%)$ \\
\hline
\end{tabular}

While analyzing the demographic profile of the study population it was observed that mean age was 54.16 years, $57.1 \%$ were male, mean duration diabetes was 12.27 years and smoking history was reported in $65.3 \%$ patients. Comorbidities observed were Hypertension (59.2\%) followed by Diabetic neuropathy ( $48.9 \% 0$ and Diabetic Retinopathy $(42.8 \%)$. 
Table 3: The sensitivity, specificity, positive predictive value (PPV), negative predicted value (NPV) and accuracy of Pulse oximetry, ABI and Combination

\begin{tabular}{|c|c|c|c|}
\hline Test & Pulse-oximetry & ABI & Combination \\
\hline Sensitivity & 82.45 & 77.13 & 93.41 \\
\hline Specificity & 93.21 & 91.46 & 97.18 \\
\hline PPV & 80.71 & 75.60 & 90.43 \\
\hline NPV & 94.32 & 84.34 & 98.11 \\
\hline Accuracy & 86.37 & 82.04 & 93.48 \\
\hline
\end{tabular}

The sensitivity, specificity, positive predictive value (PPV), negative predicted value (NPV) and accuracy of Pulse oximetry, ABI and Combination to diagnose asymptomatic PVD in diabetics was found to be $(82.45 \%, 77.13 \%$, $93.41 \%),(93.21 \%, 91.46 \%, 97.18 \%),(80.71 \%, 75.60 \%$, $90.43 \%),(94.32 \%, 84.34 \%, 98.11 \%)$ and $(86.37 \%, 82.04 \%$, $93.48 \%$ ) respectively.

\section{Discussion}

In the present study the mean age of the patients diagnosed 54.16 years with PVD. This was found in agreement with a similar previous study conducted by Kumar et al. ${ }^{[6]}$ the maximum percentage of Peripheral artery disease was seen in the 51-60 age group.

It was also observed that the Male gender and smokers group had a greater prevalence of Peripheral arterial disease. This was found in similarity with the study conducted by Kailasanadhan and Mathews consisted of 34\% smokers and 41 out 51 smokers suffered from PVD i.e. $80.4 \%{ }^{[7]}$. In another study conducted by Siao et al. reported $50 \%$ of the subjects were male, $23.1 \%$ smokers, with a mean age of 65.6 years and a mean duration of diabetes at 9.6 years ${ }^{[8]}$.

In the present investigation the major co-morbidities observed were Hypertension (59.2\%) followed by Diabetic neuropathy (48.9\%) and Diabetic Retinopathy (42.8\%). Siao et al. ${ }^{[8]}$ reported Hypertension (74.4\%) and dyslipidemia $(60.3 \%)$ were the most common comorbidities.

Chandy et al., found out 47 out of 150 patients suffering from PVD had diabetic nephropathy. This finding is in accordance with the pathogenesis model of microvascular and macrovascular complications as proposed by Fowler (2008) who held sustained hyperglycemia to be responsible for both microvascular and macrovascular complications in diabetics ${ }^{[9]}$. Association between duration of diabetes and PVD has been highlighted by a several studies reviewed by us ${ }^{[10,11]}$. Thus duration of disease seems to be an empirical risk factor supported by a large body of previous research.

The presents study revealed sensitivity, specificity, positive predictive value (PPV), negative predicted value (NPV) and accuracy of Pulse oximetry, ABI and Combination to diagnose asymptomatic PVD in diabetics was $(82.45 \%$, $77.13 \%, 93.41 \%),(93.21 \%, 91.46 \%, 97.18 \%),(80.71 \%$, $75.60 \%, 90.43 \%),(94.32 \%, 84.34 \%, 98.11 \%)$ and $(86.37 \%$, $82.04 \%, 93.48 \%$ ) respectively.

Kumar et al. ${ }^{[6]}$ in his study found sensitivity and specificity of pulse oximetry were 74.1 and $95.7 \%$ respectively, while those of ABI were $70.3 \%$ and 87.1 respectively. The PPV and NPV for pulse oximetry were $83.3 \%$ and $92.7 \%$ respectively and those for ABI were $61.3 \%$ and $91.0 \%$ respectively. Parallel testing had net sensitivity increased to $92.3 \%$ and net specificity decreased to $83.3 \% .11$

Parameswarn et al. ${ }^{[12]}$ conducted a cross-sectional study of patients with type 2 diabetes mellitus and compared the accuracy of pulse oximetry, ankle-brachial index (ABI), and a combination of the two to diagnose PVD found Pulse oximetry had a sensitivity of $77 \%$ and a specificity of $97 \%$, ABI had a sensitivity of $63 \%$ and a specificity of $97 \%$.

\section{Limitation}

The limitations of this study are as follows:

Firstly, the sample sample size and single center study would results lesser genralizabilty of the findings

Secondly, the tests were performed by single investigator, so inter observer variability would have occurred.

\section{Conclusion}

The present study concluded that Pulse-oximetry is as good as Ankle Brachial Index in the initial screening of patients with asymptomatic Peripheral Vascular Disease. It will be a perfect modest easy to-utilize potential screening device that can be utilized at the grassroots level by clinical and paramedical faculty. The blend of the two tests, that is Pulse-oximetry and Ankle Brachial Index has a much higher sensitivity and indicative precision than both of the two tests alone.

\section{References.}

1. American Diabetes Association. Peripheral arterial disease in people with diabetes. Diabetes Care. 2003; 26:3333-41.

2. Van Houtum WH, Lavery LA, Harkless LB: The impact of diabetes-related lowerextremity amputations in the Netherlands. J Diabetes Complications. 1996; 10:325-30.

3. Marinelli MR, Beach KW, Glass MJ, Primozich JF, Strandness DE Jr: Noninvasive testing vs clinical evaluation of arterial disease: a prospective study. JAMA. 1979; 241:2031-34.

4. Faglia E, Favales F, Quarantiello A, Calia P, Clelia P, Brambilla G. Angiographic evaluation of peripheral arterial occlusive disease and its role as a prognostic determinant for major amputation in diabetic subjects with foot ulcers. Diabetes Care. 1998; 21:625-30.

5. European Working Group on Critical Leg Ischaemia: Second European consensus document on chronic critical leg ischaemia. Eur J Vasc Surg 6 (Suppl. A), 1992, 1-32.

6. Kumar MS, Lohiya A, Ramesh V, Behera P, Palepu S, Rizwan SA. Sensitivity and Specificity of Pulse Oximetry and Ankle-Brachial Index for Screening Asymptomatic Peripheral Vascular Diseases inType 2 Diabetes Mellitus. Assoc Physicians India. 2016; 64(8):38-43.

7. Kailasanadhan SN, Mathews SJ. Pulse oximetry as a potential screening tool for lower extremity arterial disease in asymptomatic patients with diabetes mellitus. J Evidence Based Med Healthcare. 2018; 5(8):639-43.

8. Siao RM, So MJ, Gomez MH. Pulse Oximetry as a Screening Test for Hemodynamically Significant Lower Extremity Peripheral Artery Disease in Adults 
with Type 2 Diabetes Mellitus. JAFES. 2018; 33(2): 130-36.

9. Fowler MJ. Microvascular and Macrovascular Complications of Diabetes. Clinical Diabetes. 2008; 26:77-82.

10. Agarwal AK, Singh M, Arya V, Garg U, Singh VP, Jain V. Prevalence of peripheral arterial disease in type 2 diabetes mellitus and its correlation with coronary artery disease and its risk factors. J Assoc Physicians India. 2012; 60:28-32.

11. Garg KB, Priyanka P, Gupta A, Tak S, Sharma N, Sehra R. A Study of Ankle Brachial Index and Asymptomatic Coronary Artery Disease in Type-2 Diabetes Mellitus Patients. IOSR Journal of Dental and Medical Sciences. 2016; 15:48-51.

12. Parameswaran GI, Brand K, Dolan J. Pulse oximetry as a potential screening tool for lower extremity arterial disease in asymptomatic patients with diabetes mellitus. Arch Intern Med. 2005; 165(4):442-6. 